B eim Erlernen einer Sprache denkt man als erstes, und das meines Erachtens zu Recht, an Vokabeln und Grammatik, die ja die Grundlage bei jedem Fremdsprachenunterricht bilden. Ziel ist bei diesem letztendlich immer die Erweiterung der Sprachkompetenz in der Fremdsprache, sei es durch Hör- oder Leseverständnisübungen, durch die praktische Anwendung erlernter grammatikalischer Neuheiten oder durch andere Unterrichtsübungen, die sich zum Erreichen dieses Zieles anbieten. Jedoch kennt jeder, der eine Fremdsprache Universidad de Granada erlernt hat - bzw. erlernt, denn man lernt nie aus -, Sprechsituationen, in denen keinerlei lexikalische oder grammatikalische Fehler begangen wurden und dennoch die Kommunikation gestört ist. Ich denke hierbei an Verständigungsschwierigkeiten aufgrund eines unterschiedlichen kulturellen Hinter-
Störungsfaktoren bei der Verständigung werden. Ein einfaches Beispiel soll dies veranschaulichen: Ein Spanier ist mit irgendetwas beschäftigt und sagt zu seiner deutschen Frau: "¿Por qué no me ayudas?" und bekommt zur Antwort: "Porque no me has dicho nada", was natürlich nur zu beiderseitigem Mißmut beitragen kann. Was ist passiert? Die Frau war davon überzeugt, ihren Mann richtig verstanden zu haben, und es gab für sie keinen Zweifel, was die einzelnen Wörter der Frage bedeuteten. Nur hat sie diesen Fragesatz, wie es im Deutschen üblich ist, als Frage verstanden und daher das "por qué/warum" nicht als höfliche Aufforderung, sondern als Vorwurf gedeutet. Es war ihr folglich nicht bekannt, daß es im Spanischen als höflich gilt und es nicht ungewöhlich ist, jemanden mit einer Warum-Frage indirekt zu etwas aufzufor-

\title{
WARUM HILFST DU MIR NICHT?" ODER "HILF MIR MAL, BITTE!"? HÖFLICHKEIT IM SPANISCHEN UND DEUTSCHEN.
}

grunds, der eben in den Lehrbüchern etwas zu kurz kommt und dessen Nicht-Kenntnis oft zu Mißverständnissen zwischen Gesprächspartnern führt, von denen einer kein Muttersprachler ist und daher nicht das gleiche kulturelle Hintergrundwissen der benutzten Sprache besitzt. Ich beziehe mich in meinen Ausführungen natürlich auf das Sprachenpaar Spanisch/Deutsch bzw. auf die jeweiligen Mentalitäten.

Ein Gebiet, auf dem solche Schwierigkeiten in der Kommunikation auftreten können, sind die Umgangsformen der Höflichkeit, die in der spanischen und deutschen Kultur zwar recht ähnlich sind, sich jedoch besonders in ihren Anwendungsgraden unterscheiden und daher möglicherweise zu dern. Ein Vergleich auf der Ebene der kommunikativen Äquivalenz zeigt, wie die spanische Frage auf Deutsch hätte verstanden werden sollen:

(1) ¿Por qué no me ayudas?

(2) Hilf mir doch mal, bitte!

Es ist nicht möglich, die spanische Frage in ihrer Funktion einer höflichen Aufforderung in dieser Form ins Deutsche zu übertragen:

(3) * Warum hilfst du mir nicht? (Nicht als Aufforderung im Deutschen akzeptiert, daher Asteriskus)

Ein anderes Beispiel für unterschiedliche Umgangsformen wäre das zwanglose Grüßen. Zwei Freunde, ein Deutscher und ein Spanier, treffen sich auf der Straße und der Spanier grüßt mit "¿qué tal?/wie 


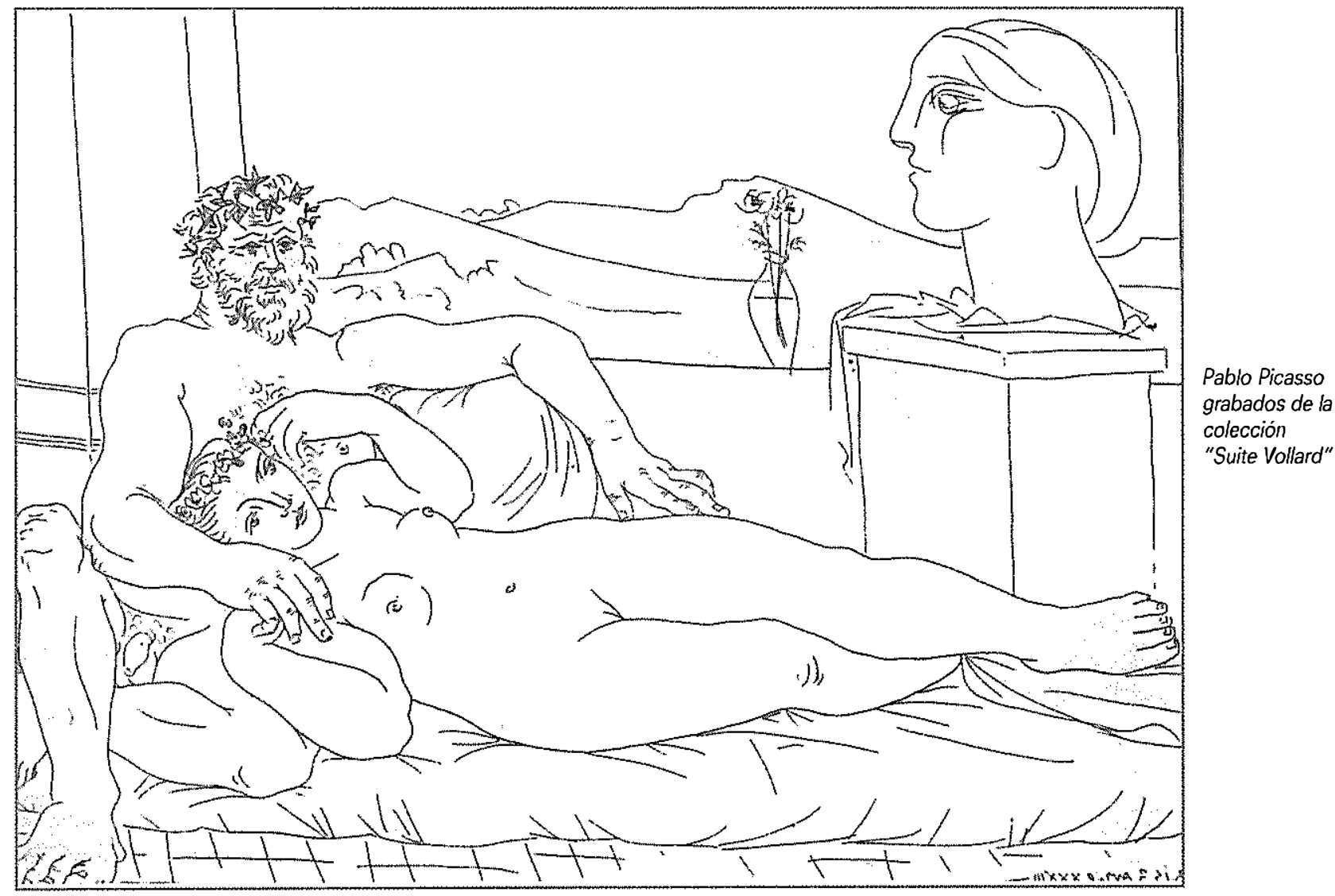

geht's?", was der Deutsche als interessierte Frage versteht und daher zum Anlaß nimmt, eine konkrete Antwort zu geben. Etwas irritiert zeigt sich dann der Deutsche, wenn er das nächste $\mathrm{Mal}$ auf seinen spanischen Feund trifft und dieser auf ein "wie geht's?/¿qué tal?" keinerlei Auskunft erteilt, sondern vielleicht eine Frage über etwas völlig anderes an ihn richtet. Dieses Beispiel zeigt, daß die spanische Begrüßungsformel "¿qué tal?" einfach ein Gruß ist und keine Frage mehr impliziert, im Gegensatz zum Deutschen, wo eben ein "wie geht's?" auch eine Frage ist. Es kann also nur von Vorteil sein, wenn man die Gewohnheiten einer anderen Kultur genauer kennt, denn dies kann unter Umständen Mißverständnisse vermeiden.

Nun ist das Feld der Höflichkeit sehr weit, und die Unterschiede zwischen Deutsch und Spanisch dürften nicht so gravierend sein wie z. B. der Unterschied zwischen Japanisch und einer europäischen Sprache, aber dennoch gibt es Situationen, in denen die Höflichkeit im Spanischen völlig anders kodofiziert wird als im Deutschen. In den weiteren Ausführungen möchte ich folgenden Fragen nachgehen:

1) Was ist unter Höflichkeit im allgemeinen zu verstehen ist und

2) wie kann es zu Mißverständnissen zwischen zwei Gesprächspartnern, deren Kulturen sich unterscheiden, kommen?

Ich behandle die Höflichkeit zuerst als Verhaltensregel und als -strategie innerhalb einer Sprachkultur, ohne auf die Schwierigkeiten einzugehen, die daraus entstehen können, daß Sprecher und Adressat zwei verschiedenen Kulturen wie im Beispiel von "¿por qué no me ayudas?" angehören. Danach wende ich mich dann dem Vergleich der Höflichkeit in beiden Sprachkulturen zu.

\section{HÖFLICHKEIT ALS VERHALTENSREGEL}

Die Sprache gilt als das wichtigste und bedeutungsvollste Mittel zur Herstellung und Aufrechterhaltung

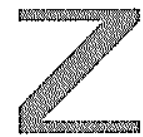




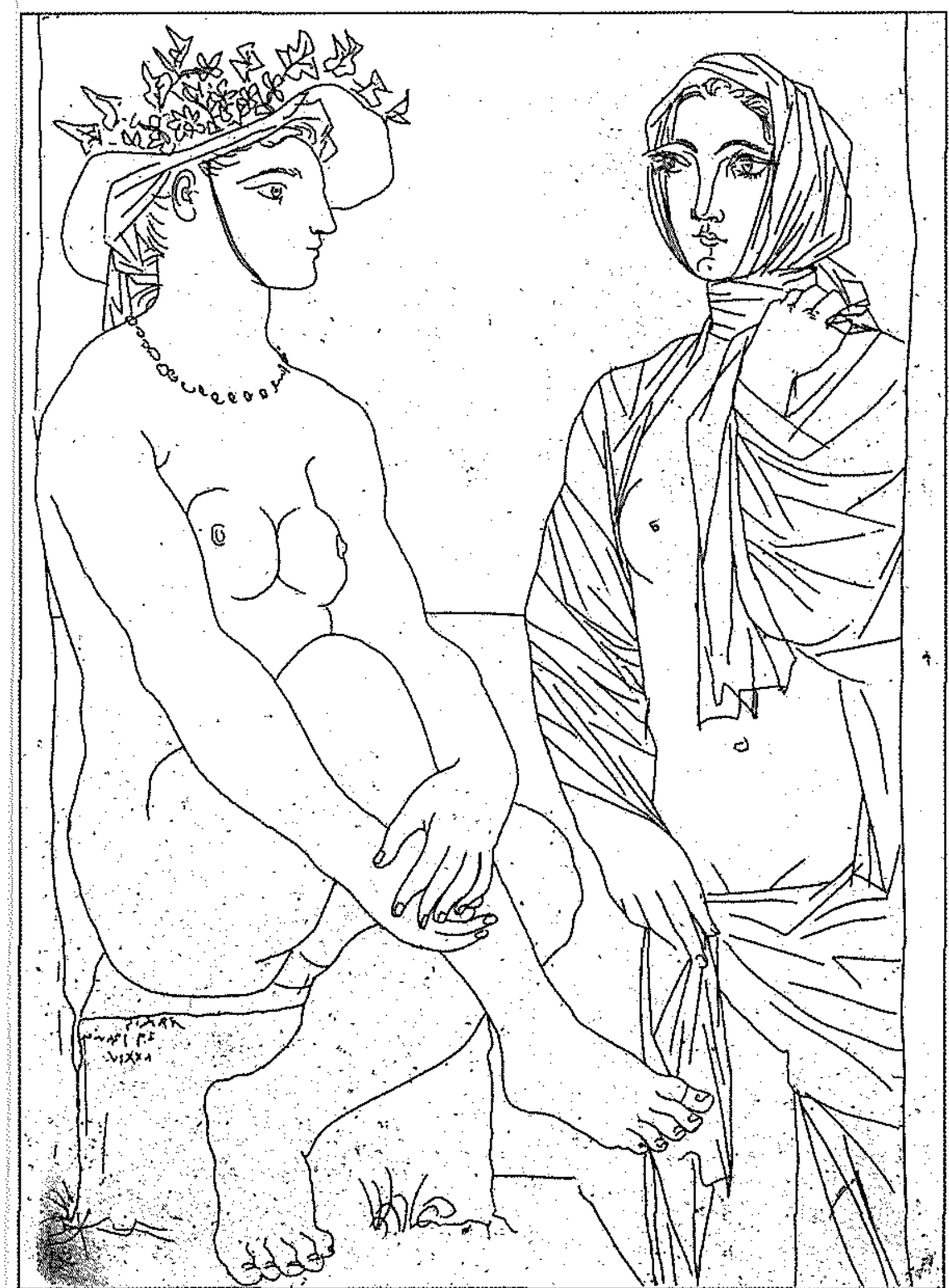

zwischenmenschlicher Beziehungen (Escandell 1993). Sie erlaubt es, miteinander in Kontakt zuu treten, Meinungen und Ansichten auszutauschen, gemeinsame Ziele zu entwerfen und zu entwickeln, ja die Sprache gilt als Mittel der Identifikation von Gruppen, Völkern und Staaten. Gerade das Zusammenleben bereitet aber auch gewisse Schwierigkeiten, und daher existieren in jeder Kultur Mechanismen, die potentielle Konflikte vermeiden helfen, worunter auch die Regeln der Höflichkeit fallen. Bei diesem sozialen Charakterzug der Höflichkeit verwundert es nicht, daß im Spanischen wie auch im
Deutschen die Wörter "cortesia" und "Höflichkeit" auf der gleichen Etymologie beruhen, denn beide verweisen auf das Leben am königlichen Hofe, wo gegen Ende des Mittelalters die Adligen begannen, sich vom Volk durch einen besonderen Verhaltenskodex abzusondern, um ihre herausragende soziale Position zu unterstreichen.

Heutzutage dienen die Formen der Höflichkeit nicht mehr dazu, soziale Positionen zu behaupten, sondern ihre Funktion liegt hauptsächlich darin, zwischenmenschliche Beziehungen zu ermöglichen und aufrechtzuerhalten. Gewisse Gesprächsabläufe sind von vorneherein festgelegt, und eine Abweichung von dieser festgelegten Struktur würde als unhöflich aufgefaßt werden. So erwartet z. B. der Patient bei einem Arztbesuch, daß inm der Arzt Fragen zu seinem Gesundheitszustand stellt, und dürfte sich kaum weigern, darauf zu antworten, geht es ja um seine eigene Gesundheit. Genauso gibt es festgelegte Regeln für eine Prüfung, und es wäre in einer Prüfung völlig unangebracht, außerhalb des festgelegten Themas Fragen zum persönlichen Wohlergehens des Studenten zu stellen.

Die beiden Beispiele belegen eine Charakteristik der Höflichkeit: bei den Normen der Höflichkeit handelt es sich um Verhaltensregeln der verbalen Kommunikation, die in einer Sprachkultur schon vorher festgelegt worden sind. Die Gesprächspartner erwarten voneinander, daß diese Verhaltensregeln auch eingehalten werden bzw. daß sie der andere Gesprächsteilnehmer kennt, was im oben angeführten Fall des Ehepaares nicht gegeben war, so daß die Kommunikation gestört war, weil es der Frau an kulturellem Hintergrundwissen fehlte und sie diese Kodifizierung der Höflichkeit nicht kannte. Wenn nun allerdings ein Mitglied einer Sprachkultur diese Regeln bewußt nicht beachtet, so bedeutet dies nicht, da $\beta$ er von einem. anderen Mitglied dieser Sprachkultur nicht verstanden werden kann, sondern nur, daß er die einschlägigen Konventionen der guten Umgangsformen sträflich verletzt und man ihn dann als sehr. unhöflich ansehen wird. Die Regeln der Höflichkeit in einer Sprachkultur sind also keine konstitutiven Regeln der Kommunikation (Searle 19903) wie zum Beispiel bestimmte formale Regeln der Grammatik, deren Nichtbeachtung dazu führen kann, daß die verbale Kommunikation erschwert, wenn nicht sogar unmöglich gemacht wird. D. h., wer die Regeln der Grammatik nicht beachtet, kann 
sich nicht verständlich machen; wer aber als Muttersprachler die Regeln der Höflichkeit nicht beachtet, schon (Haverkate 1994). Bei den Höflichkeitsregeln handelt es sich um ein Gebilde von sozialen Normen, die jede Gesellschaft für sich aufstellt und die das Verhalten ihrer Mitglieder regelt, indem sie gewisse Verfaltensformen verbietet und andere begünstigt: was diesen Normen entspricht, wird als höflich angesehen, und was sich ihnen nicht anpaßt, wird als unhöflich empfunden. Diese Verhaltensregeln sind erlernbar, da es sich um Normen handelt, die von einer Gesellschaft aufgestellt wurden. Wenn aber die Höflichkeitsregeln gesellschaftlich bedingt sind, so kann dies auch bedeuten, daß, was in der einen Kultur als höflich angesehen wird, in der anderen als ausgesprochen deplaziert gelten kann. So gilt es in der deutschen Kultur als höflich, pünktlich zu einer Einladung zu kommen, wohingegen in der französischen es die Höflichkeit geradezu verlangt, eine viertel Stunde zu spät zu kommen, um den Gastgebern genügend Zeit zu geben, auf den Besuch vorbereitet zu sein. Höflichkeit zeigt sich also in jeder Gesellschaft etwas anders bzw. kann von Kultur zu Kultur stark variieren und sogar gegensätzlich sein; dennoch folgt die Höflichkeit einem bestimmten Muster, das universal zu sein scheint; der Unterschied besteht meines Erachtens in der Anwendung dieses Handlungsmusters.

\section{STRATEGIEN DER HÖFLICHKEIT}

Die Höflichkeit ist, wie oben erwähnt, bei der Gesprächsführung zu beachten und wird besonders bei Redeakten wichtig, deren Intention darin besteht, auf den Gesprächspartner Einfluß zu nehmen und seine Entscheidungs- bzw. Handlungsfreiheit einzuschränken, besonders wenn ein Interessenkonflikt zwischen Sprecher und Adressat besteht. So muß der Sprecher bei seiner Äußerung nicht nur darauf achten, daß er seine Absichten und Ziele klar formuliert, damit diese auch verstanden werden, sondern er hat auch den sozialen Stand des Adressaten und sein Verhältnis zu ihm zu berücksichtigen. Der Sprecher wird also behutsam vorgehen und einen Balanceakt versuchen zwischen seinen Interessen und denen seines Gesprächspartners, zwischen der Durchsetzung seines Anliegens und der Erhaltung der Beziehung zum Gesprächspartner. Hier ist es besonders wichtig, die Höflichkeitsmechanismen einer Sprachkultur richtig anzuwenden. In diesem

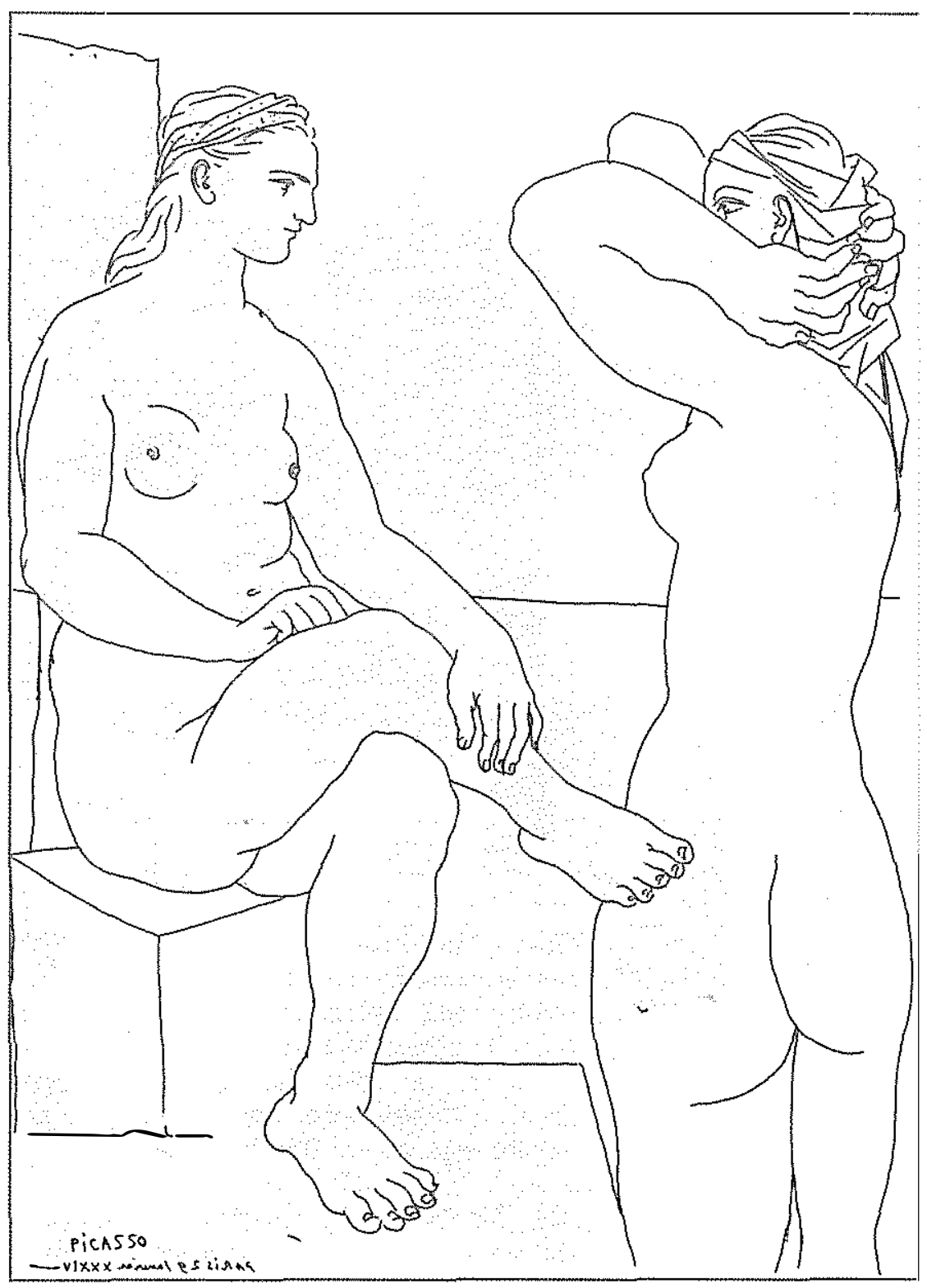

Sinne kann Höflichkeit auch als eine Verhaltensstrategie verstanden werden, die dazu dient, Interessenkonflikte zu vermeiden oder abzuschwächen (Escandell 1993). Vielleicht wäre darin der Grund zu suchen, daß Kinder die Verhaltensregeln der Höflichkeit erst recht spät erlernen, denn sie müssen einen Ausgleich zwischen verschiedenen Interessen suchen, geraten aber gleichzeitig mit etwas anderem Erlerntem in Konflikt, den Konversationsmaximen von Grice (1975), nämlich der Maxime der Qualität, der Quantität, der Relevanz und der Klarheit. Man verstößt gegen die Maxime der Qualität und der
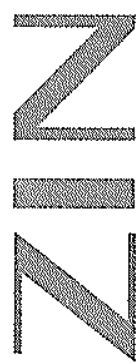


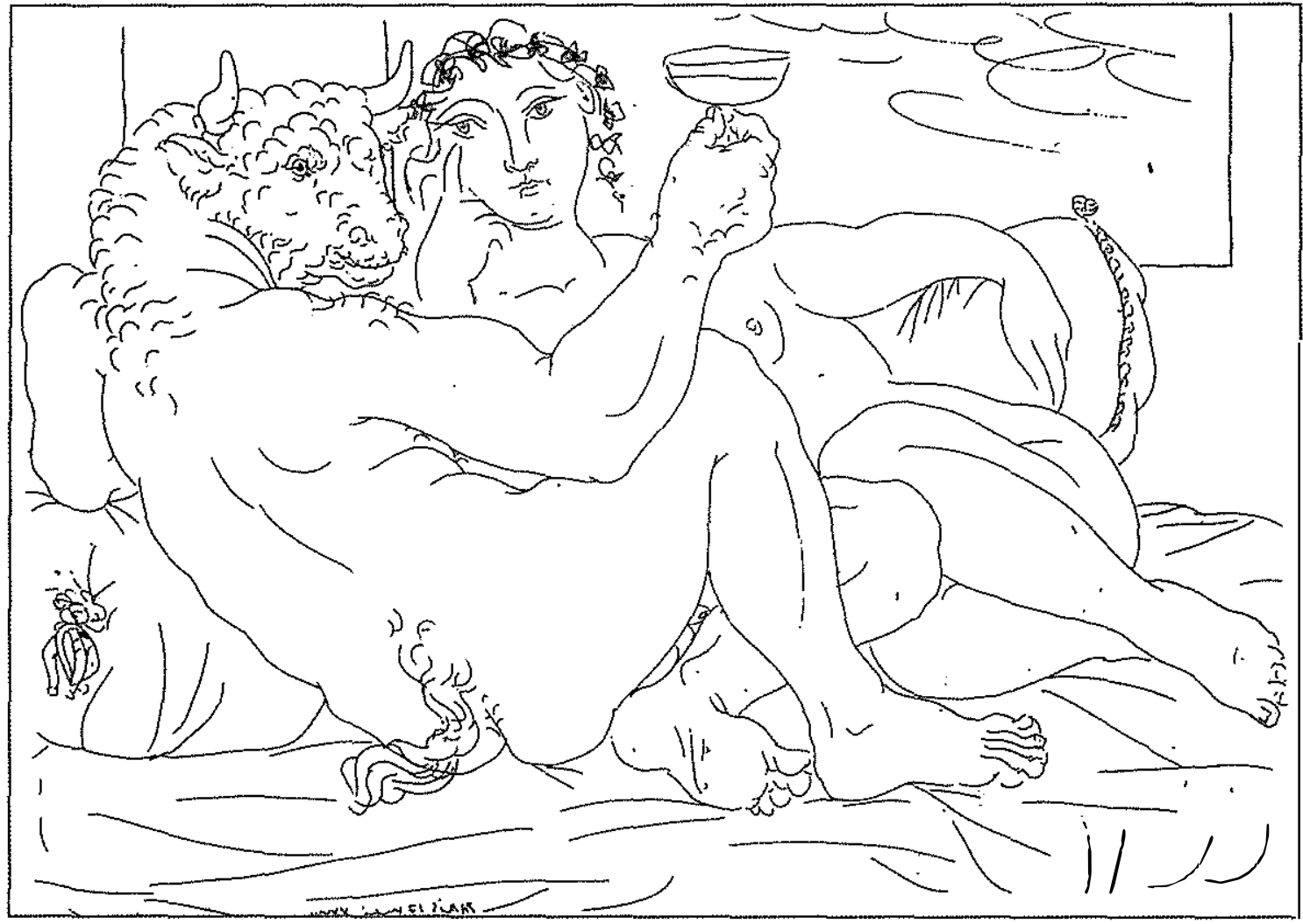

Quantität bei der Höflichkeit, wenn man die Wahrheit verschweigt, um den Gesprächspartner nicht zu verletzen oder bloßzustellen. So hat die Höflichkeit ihren Preis, denn man zahlt für sie mit gewisser Unaufrichtigkeit, Nicht-Eindeutigkeit und einem Zuviel an Worten, wobei man gleichzeitig die Konversationsmaxime von Grice mißachtet.

Die Sprachwissenschaftlerin R. Lakoff (1973) hat drei grundlegende Regeln aufgestellt, die als Gesprächsstrategien der Höflichkeit angesehen werden können:

1) Zwinge deinem Gesprächspartner nicht deinen Willen auf!

2) Biete Alternativen!

3) Achte darauf, daß dein Gesprächspartner sich wohl fühlt; sei freundlich!

Jede Regel bezieht sich auf ein bestimmtes Anwendungsgebiet entsprechend dem zwischen den Gesprächspartnern bestehenden Verhältnis, das vom Grad der Vertrautheit oder der Formalität der Kommunikationssituation abhängt:
Regel 1) wäre besonders in Situationen zu beachten, wo zwischen den Gesprächsteilnehmern eine klare soziale Distanz vorliegt oder keinerlei Vertrautheit vorhanden ist, $d$. h. wenn die Gesprächssituation einen formellen Charakter hat. Keiner der Gesprächspartner darf den anderen in irgendeiner Form dazu drängen, etwas zu tun. In diesem Fall besteht die Strategie der Höflichkeit darin, jeden möglichen Zwang zu vermeiden, indem man mit indirekten Formeln um Erlaubnis fragt:

(4) Dürfte ich Sie fragen, wenn es Ihnen nichts ausmacht, ob ich mal einen Blick in Ihre Zeitung werfen könnte?

Bei dieser Strategie werden eine direkte Ausdrucksweise und eigene Meinungen vermieden und man zieht eine allgemeine Sprache vor ( $\mathrm{vgl}$. Beispielsatz (5) und im Gegensatz dazu (6), der sehr direkt ist und nicht als höflich gelten kann):

(5) Bei diesem Wetter ist es gut, wenn man eine Garage hat; man braucht dann sein Auto nicht so oft zu putzen. 
(6) Waschen Sie mal wieder Ihr Auto!

Regel 2) bezieht sich auf Gesprächssituationen, in denen Sprecher und Adressat sozial gleichgestellt sind, ohne daß jedoch ein besonderes Vertrauensverhältnis zwischen ihnen bestünde. Alternativen zu bieten, bedeutet, sein Anliegen so vorzubringen, daß eine Ablehung nicht zu einem Konflikt führt.

(7) Könntest du mir dieses Buch mal leihen oder brauchst du es gerade selbst?

Schließlich dient Regel 3) dazu, die sozialen Bindungen zum Adressaten zu verstärken, indem man sich für seine Angelegenheiten interessiert und inm seine Position recht angenehm macht. Hier gebraucht man vor allem eine persönliche Audrucksform unter Verwendung von Personalpronomen, und häufig bezieht sich der Sprecher in seiner Aufforderung auch auf sich selbst, um eine Identifikation mit seinem Gesprächspartner herzustellen.

(8) Welchen Film schauen wir uns heute abend an?

Die Regeln 1) und 2) treten vor allem bei Sprechakten auf, die zur Ermahnung oder Aufforderung bestimmt sind, und verfolgen die Maxime, darauf zu achten, daß der Gesprächspartner sich nicht angegriffen fühlt; denn der Sprecher, der eine Aufforderung ausspricht, dringt in den Persönlichkeitsbereich des Gesprächspartners ein und will diesen zu einer Handlung bringen, die in seinem eigenen Interesse liegt. Wenn also der Sprecher die Regeln 1) und 2) beachtet, so gibt er seinem Gesprächspartner indirekt zu verstehen, daß er sich bewußt ist, ihn in seiner Handlungsfreiheit einzuschränken; so greift er zu einer Bitte anstatt zu einer Befehlsform.

(9) Würde es dir etwas ausmachen, wenn du mir dein Auto leihst?

Die Regel 3) dient dagegen vor allem dazu, dem Gesprächsteilnehmer das Gefühl zu vermitteln, daß man inn als eine nette Person ansieht und gerne mit ihm zusammen ist
(Escandell 1993).

Eine andere Art, die Höflichkeit zu erklären, bestünde in den Ausführungen von Brown und Levinson (1987), die von der Vorstellung ausgehen, daß sich jede Person im Alltag zu behaupten hat und deshalb immer wieder mit seinen Mitmenschen in Konflikt gerät. Höflichkeit erlangt in diesem Zusammenhang die Funktion, eine Eskalierung derartiger Konflikte zu vermeiden, indem sie jeweils das "Gesicht" der Gesprächsteilnehmer wahren hilft. Brown und Levinson führen somit "Gesicht" ("face", "cara") als terminus technicus ein und beziehen sich damit auf die zwei Seiten der Höflichkeit, die negative und die positive. Das "negative Gesicht" stellt einen Angriff auf die Intimsphäre, die Entscheidungs- und Handlungsfreiheit des Adressaten dar bzw. die Ablehnung seiner Willensäußerung; das "positive Gesicht" hingegen bezieht sich auf die positive Vorstellung, die einjeder von sich selbst hat und die er natürlich bewahren will und von den anderen bestätigt bzw. respektiert haben will. Bei der Höflichkeit geht es also immer darum, das "Gesicht", sei es das des Sprechers oder das des Adressaten, bei Sprechakten zu wahren, die eine Gesichtsbedrohung ("face-threatening") darstellen. Um diese potentielle Bedrohung abzuschwächen, bedient man sich der Höflichkeit.

Der Grad der Höflichkeit hängt nun im Grunde nicht nur von den drei Regeln Lakoffs ab, sondern folgt auch den Ansichten, die der Sprecher von der Ange-

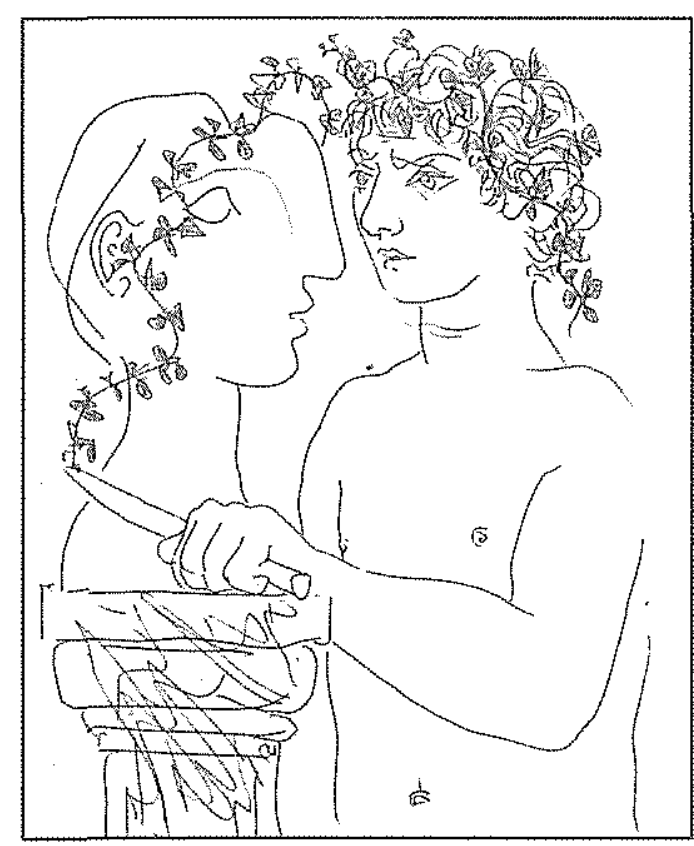
messenheit der Höflichkeit in einer bestimmten Situation hat. So stellt sich hierbei immer die Frage, wessen Interesse wird dabei berücksichtigt, wenn eine Bitte, eine Ermahnung,ein Befehl, ein Rat oder eine Aufforderung befolgt wird. Wenn eine indirekte höfliche Aufforderung im Interesse des Adressaten liegt, dann ist die Aussageform klarer und kürzer, als wenn der Sprecher in seinem eigenen Interesse versucht, auf seinen Gesprächspartner Einfluß zu nehmen:
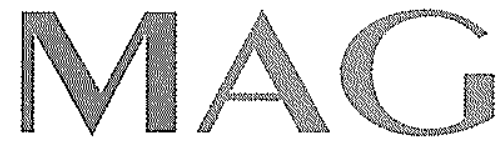
(10) Mach dir mal Licht! Du willst doch lesen.

(11) Ich möchte etwas lesen. Könntest du mal das Licht anmachen? Es ist so dunkel hier.

In Beispielsatz (10) wird der Imperativ mit der abschwächenden Modalpartikel "mal" verwendet; es handelt sich jedoch nicht um eine unhöfliche Aufforderung, da es ja im Interesse des Hörers liegt, wenn das Licht angeschaltet wird. Dagegen hat Satz (11) den Nutzen des Sprechers zum Anlaß und gebraucht daher einen indirekten Stil unter Verwendung von Modalverben ("möchte" und "könnte") und einer Frage statt einer direkten Aufforderung. So erscheint er schon der Form nach höflicher. Und noch etwas läßt sich aus den letzten Beispielsätzen zur Höflichkeit herleiten: der Grad an Höflichkeit eines Sprechaktes kann nicht isoliert gemessen werden; es kommt immer darauf an, in welchem Kontext und in welcher Sprechsituation die Äußerung fällt, und der sprachliche Aufwand hängt davon $a b$, wem sie nützt. Liegt der Nutzen beim Adressaten, so ist der sprachliche Aufwand geringer, als wenn er beim Sprecher liegt. Darüberhinaus läßt sich sagen, daß der Grad der Höflichkeit noch von folgenden Faktoren bestimmt wird (Schmelz 1993):

1) je größer der soziale Abstand zwischen Sprecher und Adressat, umso größer das $\mathrm{Ma} B$ an Höflichkeit;

2) je größer die Macht des Adressaten über den Sprecher, umso größer das Maß an Höflichkeit;

3) je größer der Grad des Drucks, der ausgeübt wird, umso größer das $\mathrm{Maß}$ an Höflichkeit.

Dies möge nun zur allgemeinen Beschreibung der Strategien der Höflichkeit genügen und es ist hoffentlich deutlich geworden, daß die Höflichkeit an sich eine entscheidende Rolle in den zwischenmenschlichen Beziehungen spielt.

\section{HÖFLICHKEIT IM SPANISCHEN UND DEUTSCHEN}

Es dürfte nun interessant sein, einzelne Beispiele der Höflichkeit im Spanischen und Deutschen anzuführen, die deutlich machen, wo Gemeinsamkeiten und Unterschiede zwischen beiden Kulturen liegen.

Leider gibt es kaum Literatur, die dieses Thema ausführlich behandelt. In diesem Zusammenhang wäre jedoch das Buch von Beinhauer El español coloquial zu erwähnen, das allerdings den Nachteil hat, daß es schon größtenteils veraltet ist. So muß ich mich bei den folgenden Beispielen und ihrer Analyse auch auf eigene Erfahrungen stützen.

Ganz allgemein ist als erstes festzuhalten, daß sich das Deutsche normalerweise formellerer Ausdrucksweisen bedient als das Spanische bzw. das Andalusische. So ist es nahezu obligatorisch im Deutschen, bei der Anrede einer fremden, erwachsenen Person die Höflichkeitsform "Sie" zu verwenden, es sei dennman befindet sich in Gesellschaft von gleichaltrigen Jugendlichen oder Studenten; aber selbst da ist es eher zu empfehlen: im Zweifelsfalle immer "Sie". Hintergrund dieser Verhaltensform wäre die genannte Regel 1), die bei sozialer Distanz zu beachten ist. Im Spanischen wird sie nicht so strikt gehandhabt, und anscheinend verwendet man die Höflichkeitsanrede "usted" nur in der Kennenlernphase, um schnell zum vertraulicheren "tú" überzuwechseln. Auf der gleichen Ebene wie die Höflichkeitanrede "Sie" liegt im Deutschen auch der Gebrauch von "bitte" und "danke". Es gilt als höflich, diese Wörter bei jedem Anlaß zu verwenden, sei es beim Einkaufen von Brötchen, dem Fragen nach dem Weg oder der Uhrzeit oder dem Einreichen von irgendwelchen Formularen bei einem Beamten.

Auffallend werden die Unterschiede dann bei der Vorgehenweise in beiden Sprachkulturen, wenn es sich darum handelt, jemanden um einen Gefallen zu bitten. Hier ist darauf hinzuweisen, daß im Deutschen einer zwar direkten, aber doch höflichen Aussage immer der Vorzug zu geben ist. In der spanischen Sprachkultur gilt es als unschön, bei einem Gespräch mit einem Bekannten oder einem Freund gleich auf den Punkt zu kommen und zu sagen, warum man zum Beispiel anruft. Es ist nahezu ein Ritual einzuhalten, bei dem man sich erst nach dem allgemeinen Befinden des Gesprächspartners und 
eventuell seiner Familie erkundigt, bevor man eigentlich nur so nebenbei und anscheinend zufällig auf den eigentlichen Grund des Anrufs kommt. Hier steht folglich die Regel 3) im Vordergrund, nämlich dem Gesprächspartner gegenüber freundlich zu sein und nicht gleich mit der Tür ins Haus zu fallen. Der Deutsche empfindet da anders; es könnte dabei die Regel formuliert werden: "Lieber kurz und bündig und so wenig wie möglich gegen die Grundsätze von Grice verstoßen." D. h. nach einem kurzen Gruß kommt man am Telephon gleich zur Sache und danach kommt man dann vielleicht zu Dingen, die in der spanischen Sprachkulur als erstes behandelt werden.

(12) Hallo, Peter! Ich bin's, Ralph. Du, könnte ich mir vielleicht deinen Schlafsack ausleihen, ich möchte nämlich übers Wochenende an den Bodensee zelten gehen.

Satz (12) wäre ein Beispiel für den Anfang eines ungezwungenen Telephongesprächs zwischen zwei Freunden, was im Spanischen so wohl kaum vorkommen dürfte, da die Vorgehensstrategie viel zu offen und direkt wäre, auch wenn es sich um Freunde handelt. Der Grund dürfte darin liegen, daß es im Deutschen eher akzeptiert wird, eine Gesichtsbedrohung zuzulassen, oder man eben der Konversationsmaxime der Klarheit und Quantität den Vorrang einräumt.

Ich glaube in diesem Zusammenhang an den grundlegenden Unterschied zwischen den spanischen und deutschen Höflichkeitsformen gekommen zu sein. Im Gegensatz zur deutschen Sprachkultur folgt die spanische Höflichkeit nach Möglichkeit vor allem der Regel 3) von Lakoff, die besagt:

3) Achte darauf, daß dein Gesprächspartner sich wohl fühlt; sei freundlich!

Die deutsche Mentalität bewegt sich dagegen mehr in den Bereichen der Regeln 1) und 2):

1) Zwinge deinem Gesprächspartner nicht deinen Willen auf!

2) Biete Alternativen!

Darüberhinaus hat sie die Tendenz, die Griceschen Konversationsmaxime der Quantität und der Klarheit möglichst zu beachten (was nicht bedeuten soll, daß dies auch immer so ist oder sein muß), wogegen das Spanische mehr dazu neigt, die zwei Grundsätze von Grice nicht zu berücksichtigen. So erklärt sich im

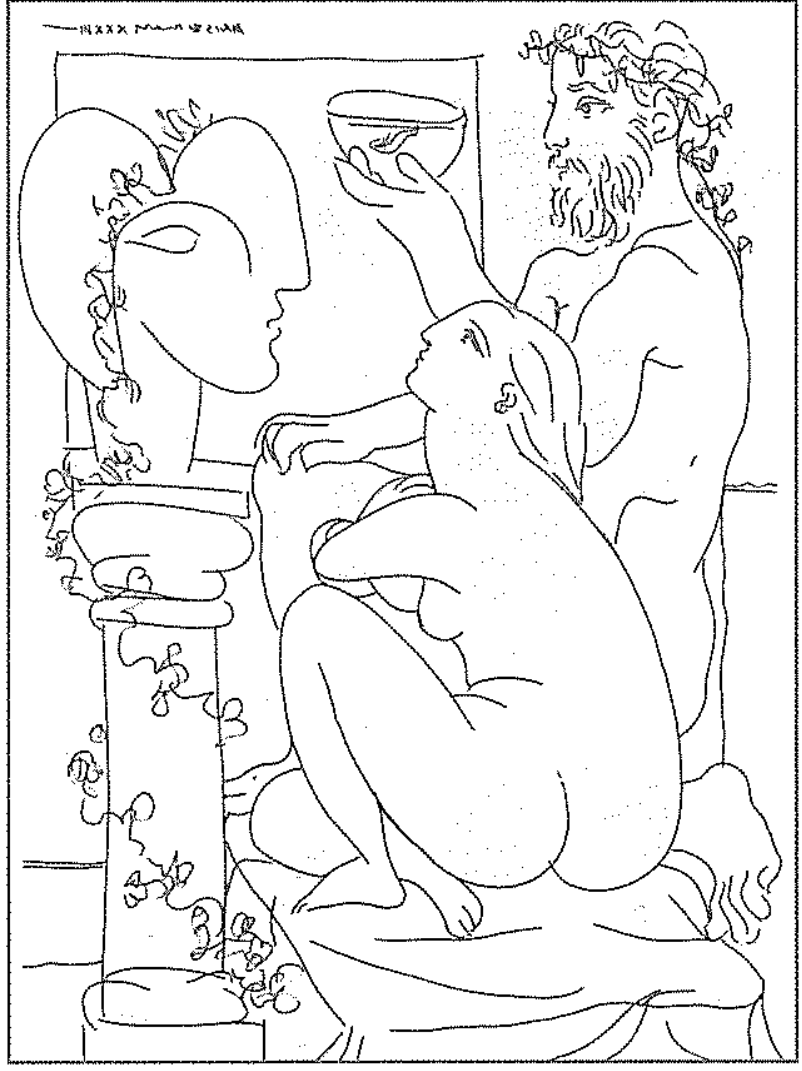

Deutschen der häufige Gebrauch von "bitte", das je nach Kommunikationssituation verschiedene Bedeutungen annehmen kann und dann den zweckorientierten Charakter der deutschen Mentalität deutlich werden läßt:

(13) Beim Friseur kommt ein neuer Kunde an die Reihe und der Friseur sagt nur: "Bitte schön!", was als höfliche Aufforderung gilt.

Im Spanischen wäre dies weniger üblich, allerdings kommt es häufiger zur typischen Aufforderung (14) "¡Cuando quiera!", die eben dem Kunden einen weiten Raum der Entscheidungsfreiheit zugesteht und ihm alle Möglichkeiten offenhält. Im Deutschen würde diese Art von Höflichkeit eher als negativ angesehen und könnte zur offenen Ablehnung führen, da sie zu unterwürfig erscheint.

Eine weitere Situation, wo die Anwendung der Höflichkeitsform aus der anderen Kultur zu Mißstimmungen führen kann, wäre der Augenblick des Zahlens von Trinkgeld. Kurioserweise kommt hier das gleiche Prinzip zur Geltung, das der Regel 3), nur wird es jeweils auf den anderen Gesprächsteilnehmer ange- 


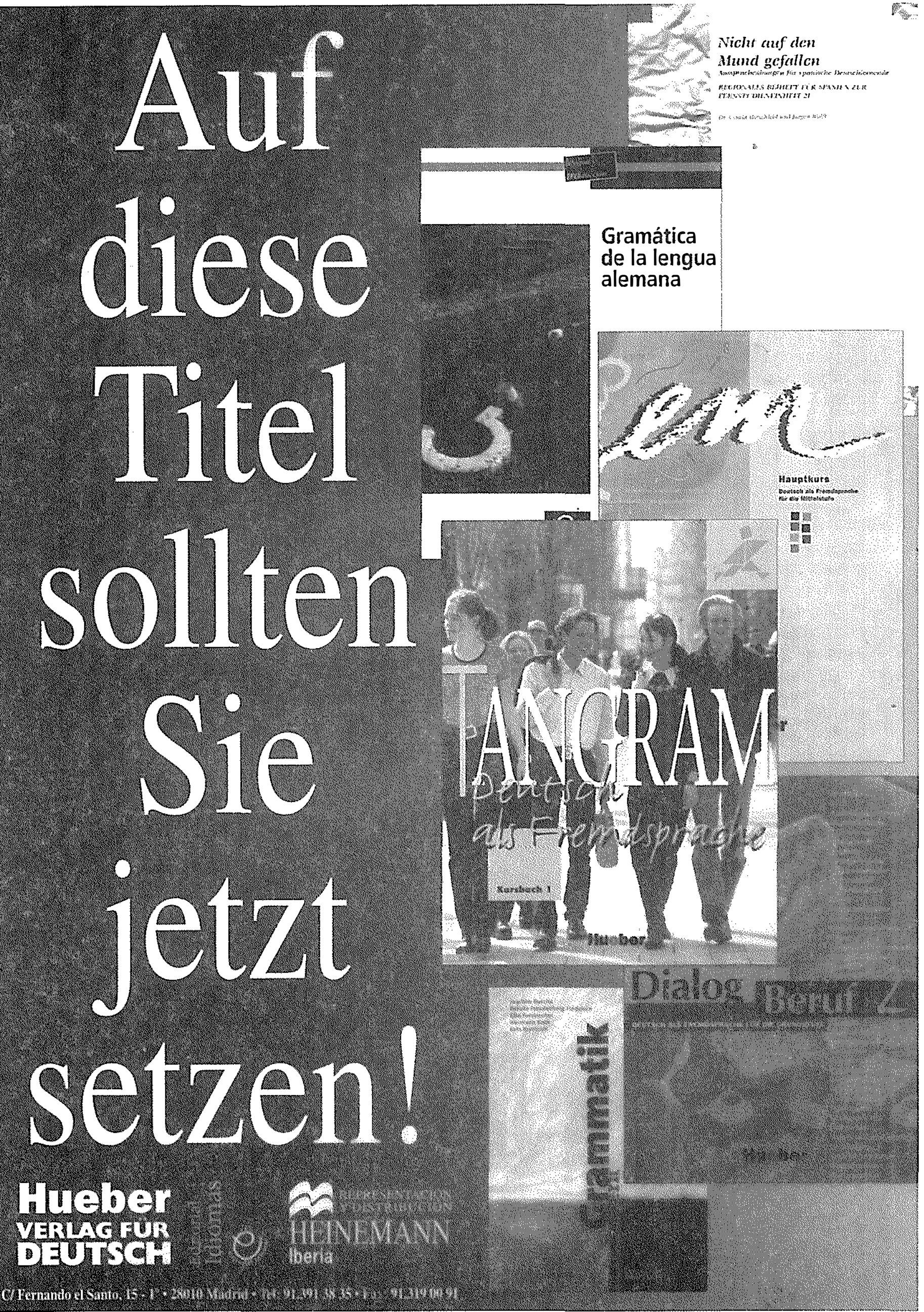


wendet, einmal auf den Gast und das andere Mal auf den Kellner. In Spanien legt man sein Trinkgeld, nachdem man gezahlt hat, auf den Tisch und der Kellner erwartet es auch so, weil er ja höflich ist und dem Gast in keinster Weise irgendwie dazu drängen möchte, sondern ja will, daß der Gast sich wohl fühlt (Regel 3). Der Kunde entscheidet sich frei und ungezwungen zu einer Anerkennung des geleisteten Dienstes. Im deutschen Kulturkreis dagegen sieht man eher ärgerliche Gesichter bei den Kellnern, wenn man den zu zahlenden Betrag nicht gleich aufrundet, sich erst das Rausgeld geben läßt und dann das Trinkgeld auf den Tisch legt. Zwar heißt es immer, der Kunde ist König, aber dennoch fühlt sich auch der Gast zur Höflichkeit verpflichtet und versucht, dem Kellner das Leben bzw. seine Arbeit so einfach und leicht wie nur möglich zu machen, und dazu gehört offensichtlich auch, das Trinkgeld gleich mit der Rechnung zu zahlen (Regel 3: sei freundlich!).

\section{ZUSAMMENFASSUNG}

Die primäre Funktion und Bedeutung der Höflichkeit liegt darin, die sozialen Beziehungen zu pflegen, zu stärken und zu fördern. Hierzu bedient sich eine jede Sprachkultur eines ihr eigenen Regelsystems, mit dem jedes Gesellschaftsmitglied von Kind an vertraut gemacht wird, worin dann auch der Grund zu sehen ist, warum es so schwierig ist, sich einem neuen Verhaltenskodex anzupassen, da ja der muttersprachliche stark verinnerlicht ist und nicht einfach abgelegt werden kann.

Anhand dieser Untersuchung konnte festgestellt werden, worin die grundlegenden allgemeinen oder universalen Verhaltensweisen, die der Höflichkeit zugrundeliegen, bestehen. Hierzu waren die Regeln von Lakoff äußerst nützlich, konnten sie doch erklären, nach welchen Kriterien sich einzelne Strategien der Höflichkeit richten, nämlich keinen Druck auf den Gesprächspartner auszuüben, ihm Auswahlmöglichkeiten anzubieten und ihn in seinem Selbstverständnis zu bestärken.

Es wurde auch deutlich, daß diese Regeln eigentlich im Gegensatz zu den Maximen von Grice stehen und sich daraus gewisse Unterschiede bei der Anwendung von Höflichkeitsformen im Deutschen und Spanischen erklären lassen. Generell kann gesagt werden, daß die deutsche Sprachkultur mehr den Grundsatz der Klarheit und der Quantität berücksichtigt als die spanische, die eher dazu tendiert, vor allem die dritte Regel Lakoffs zu beachten. Im Extremfall, wie beim Beispiel vom Trinkgeld näher dargelegt wurde, können die Höflichkeitsformen sich völlig konträr gegenüberstehen, so daß eine Höflichkeitsform in einem ihr fremden Kulturkreis nahezu in ihr Gegenteil umschlagen kann

Es wäre wünschenswert, wenn das vernachlässigte Gebiet der unterschiedlichen Verhaltensweisen, Gebräuche und Sitten, die alle eine bestimmte Mentalität prägen und ihren Niederschlag in der ihr zugehörenden Sprache finden, aus seinem stiefmütterlichen Dasein entlassen würde und nicht nur Eingang in theoretischen Abhandlungen, sondern auch in die Unterrichtsbücher der Fremdsprachen fände und so den ihm gebührenden Platz einnähme.

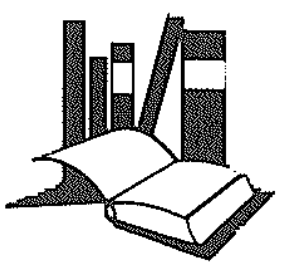

L.J

\section{LITERATURVERZEICHNIS}

BEINHAUER, W. (1985). El español coloquial. Madrid: Gredos.

BROWN, P. \& S. LEVINSON (1978). Politeness. Some Universals in Language Use. Cambridge: University Press.

ESCANDELL MDAL, M.V. (1993). Introducción a la pragmática. Barcelona: Anthropos; Madrid: UNED.

GRICE, H. P. (1975). "Logic and Conversation." COLE, P. \& J. L. MORGAN (Hrsg.). Syntax and semantics. Bd. 3: Speech Acts. Nueva York: Academic Press, 41-58.

HAVERKATE, H. (1994). La cortesia verbal. Madrid: Gredos.

LAKOF, R. (1973). "The Logic of Politeness, or Minding your P's and Q's." Proceedings of the Ninth Regional Meeting of the Chicago Linguistic Society, 345-356.

SCHMELZ, M. P. (1993). Psychologie der Höflichkeit. Frankfurt a. M.: Peter Lang.

SEARLE, J. (19903). Actos de habla. Madrid: Cátedra.
} 